\title{
Estrutura morfológica do fígado de tambaqui Colossoma macropomum (Cuvier, 1818) ${ }^{1}$
}

\author{
Gerlane de Medeiros Costa ${ }^{2 *}$, Ricardo Claro Ortis ${ }^{3}$, Mendelson Guerreiro de Lima², \\ Juliana Barbosa Casals ${ }^{4}$, Ana Rita de Lima ${ }^{5}$ e José Roberto Kfoury Jr ${ }^{6}$
}

\begin{abstract}
Costa G.M., Ortis R.C., Lima M.G., Casals J.B., Lima A.R. \& Kfoury Jr J.R. 2012. [Morphological structure of the liver in tambaqui, Colossoma macropomum (Cuvier, 1818).] Estrutura morfológica do fígado de tambaqui Colossoma macropomum (Cuvier, 1818). Pesquisa Veterinária Brasileira 32(9):947-950. Laboratório de Anatomia Animal, Departamento de Ciências Biológicas, Universidade do Estado do Mato Grosso, Campus de Alta Floresta, MT 78580-000, Brazil. E-mail: gerlanemcosta@yahoo.com.br

This research aimed to describe the macroscopic and microscopic liver of tambaqui, Colossoma macropomum, Teleost freshwater Family Characidae, of great economic interest for the Amazon basin. We used six juveniles aged between six month and one year, from the small holding Esteio, Alta Floresta/MT, that develops mainly fish farming. The body was photographed in situ, described macroscopically, and fragments were removed and processed by routine histological techniques through paraffin embedding and HE staining. The liver, located ventrally to the swim bladder and craniodorsally to the stomach, is brownish red and consisted of three lobes, the right lateral, the left lateral and the ventral lobe. Microscopically, the parenchyma consists of hepatocytes varying from irregular rounded hexagonal to round forms with a large and central nucleus, and arranged in linear strings limited by sinusoids and radiating to central veins, but with absence of liver lobules. The central veins are distributed throughout the parenchyma, while the portal space consists in most cases only of a hepatic vein and bile duct; elsewhere exist artery and duct. Formation of portal triads was not founde. Melano macrophages were frequently seen dispersed throughout the central parenchyma. The morphofunctional study of the digestive system of fishes of the Amazon basin is important to obtain knowledge about their weight gain, large scale production for human consumption and preservation of the species, and has also its importance for being used as bioindicators today.
\end{abstract}

INDEX TERMS: Tambaqui, Colossoma macropomum, fish, liver, morphology, teleostei.

RESUMO.- Este trabalho teve como objetivo descrever macro e microscopicamente o fígado do Tambaqui Colossoma macropomum, Teleósteo de água doce da Família Characi-

\footnotetext{
${ }^{1}$ Recebido em 26 de dezembro de 2011.

Aceito para publicação em 20 de junho de 2012.

${ }^{2}$ Faculdade de Licenciatura em Ciências Biológicas, Universidade do Estado do Mato Grosso (Unemat), Rodov. MT $208 \mathrm{Km} 143$ s/n, Bairro Jardim Tropical, Alta Floresta, MT 78580-000, Brasil. *Autor para correspondência: gerlanemcosta@yahoo.com.br

${ }^{3}$ Graduado em Ciências Biológicas, Unemat, Alta Floresta, MT.

${ }^{4}$ Pós-Graduanda, Universidade de São Paulo (USP), Pirassununga, SP 13635-000, Brasil.

${ }^{5}$ Instituto de Saúde e Produção Animal (ISPA), Faculdade de Medicina Veterinária, Universidade Federal Rural da Amazônia (UFRA), Av. Pres. Tancredo Neves 2501, Bairro Montese, Belém, PA 66077-530, Brasil.

${ }^{6}$ Faculdade de Medicina Veterinária e Zootecnia (FMVZ), USP, Av. Prof. Dr. Orlando Marques de Paiva 87, Cidade Universitária, São Paulo, SP 05508-270, Brasil.
}

dae, de grande interesse econômico da bacia Amazônica. Foram utilizados seis (6) exemplares jovens com idade entre seis meses e um ano, oriundos da Chácara Esteio, Alta Floresta, MT, que desenvolve principalmente a prática da piscicultura. 0 órgão foi fotodocumentado in situ e descrito macroscopicamente, em seguida procedeu-se a retirada de fragmentos deste, que foram processados pelas técnicas histológicas rotineiras para inclusão em parafina e coloração de HE. 0 fígado localizou-se ventral à bexiga natatória e craniodorsalmente ao estômago, apresentou coloração amarronzada a vermelho, constituído por três lobos hepáticos, o lobo lateral direito, o lobo lateral esquerdo e o lobo ventral. Microscopicamente, o parênquima era constituído por hepatócitos com formato que variou do arredondado irregular hexagonal ao redondo com núcleo grande e central, arranjados em cordões lineares limitados por sinusóides que irradiam para veias centrais, e com ausência de lóbu- 
los hepáticos. As veias centrais estavam distribuídas pelo parênquima, enquanto que o espaço porta, na maioria das vezes, era constituído apenas por uma veia hepática e o ducto biliar, em outros locais foi observado, uma artéria e um ducto. Não foi observada a formação de tríades portais. Foram frequentemente observados melano macrófagos centrais dispersos pelo parênquima. 0 estudo morfofuncional do Aparelho Digestório de peixes da bacia Amazônica, se faz pertinente com vistas ao conhecimento do aproveitamento de ganho de peso e produção em alta escala para consumo humano e preservação da espécie, além da importância de estarem sendo utilizados como bioindicadores atualmente.

TERMO DE INDEXAÇÃO: Tambaqui, Colossoma macropomum, morfologia, neotropical, peixe, teleósteo.

\section{INTRODUÇÃO}

Os rios que formam a bacia amazônica retêm a maior biodiversidade íctia de água doce do planeta (Vari \& Malabarba 1998, Santos \& Ferreira 1999) e grande parte desta diversidade ainda é pouco conhecida no que diz respeito a sua morfologia (Bicca et al. 2006, Arandas et al. 2009).

Endêmico da bacia Amazônica, o tambaqui é um peixe pertencente à família Characidae e é considerado o maior dos Characiformesendo. Uma das espécies mais desejadas pelos pescadores, por ser forte, apresentar carne de excelente sabor, com pouca espinha. Na natureza, alcança porte máximo entre $90 \mathrm{~cm}$ a $100 \mathrm{~cm}$ de comprimento, com peso máximo entre $30 \mathrm{~kg}$ e $40 \mathrm{~kg}$ (Goulding \& Carvalho 1982, Goulding 1993). Com uma alta adaptabilidade aos ambientes de cultivo é responsável por uma considerável parcela do mercado regional de pescado, atraindo um número expressivo de pesquisadores para estudar e propor meios de reduzir os custos e maximizar a sua produção para melhor atender ao mercado pesqueiro (Arbeláez-Rojas et al. 2002).

0 fígado de peixes é um órgão compacto, localizado ventralmente na cavidade celomática. Seu tamanho, forma e volume estão adaptados ao espaço utilizado pelos outros órgãos viscerais e varia muito entre as espécies. Apresenta uma coloração vermelho-pardo amarronzado (Bruslé \& Anadon 1996) ou avermelhado, ocasionado pela densa vascularização, tendendo para o amarelo, quando o estoque de gordura é alto (Ostrander 2000).

Os peixes vêm sendo amplamente utilizados para avaliar a saúde de ecossistemas aquáticos e as modificações fisiológicas ocasionadas, atuando como bioindicadores de poluição ambiental (Kock et al. 1996). Na Amazônia o tambaqui tem sido utilizado como bioindicador e biomonitor de agentes químicos, físicos e biológicos (Brust \& Pinto 2005, Araújo et al. 2005, Oliveira et al. 2005).

Observando a importância econômica e ambiental desta espécie, este trabalho teve como objetivo descrever a morfologia do fígado do Tambaqui, Colossoma macropomum, oferecendo uma base de informações das estruturas normais do órgão descrito.

\section{MATERIAIS E MÉTODOS}

Para a avaliação das estruturas morfológicas do fígado de tambaqui, foram utilizados seis exemplares juvenis, com idade entre seis meses e um ano, oriundos da Chácara Esteio, Alta Floresta/MT, que tem como principal atividade a prática da piscicultura.

Os animais foram coletados com auxílio de uma rede de arrasto, colocados em recipientes plásticos, anestesiados em solução de benzocaína a 50ppm em solução aquosa (1 $\mathrm{g}$ de benzocaína em 20L de água) e eutanasiados. Após completa paralização dos movimentos operculares, foi feita uma abertura da cavidade abdominal por meio de incisão ventral, obtendo-se uma janela através da qual o órgão foi fotodocumentado in situ com o uso de máquina digital.

Após a extração do órgão, pequenos fragmentos foram obtidos e fixados em solução de paraformaldeído $10 \%$ para realização da microscopia de luz, processados no Laboratório de Histotécnicas do Centro Universitário da Fundação de Ensino Octavio Bastos, UNIFEOB, São João da Boa Vista, SP, segundo a técnica de Behmer (1976), em séries crescentes de álcoois, até o álcool absoluto e em seguida incluído em paraplast. Posteriormente, os fragmentos foram cortados com o uso de micrótomo com espessura de $4 \mu \mathrm{m}$ e corados pelas hematoxilina e eosina (HE).

\section{RESULTADOS}

O fígado foi a maior glândula encontrada na cavidade celomática de Colossoma macropomum, localizando-se ventral à bexiga natatória e craniodorsalmente ao estômago, com coloração vermelho amarronzada. Macroscopicamente era constituído por três lobos: os lobos hepáticos direito e esquerdo e o lobo hepático ventral (Fig.1). A vesícula biliar constituía-se de um divertículo (saculação) na região posterior, ou face interna, do lobo hepático ventral, apresentando um ducto que atravessava toda a cavidade celomática e terminava na região caudal em forma de vesícula, tendo sido dividida em região cranial, média e caudal, e apresentando conteúdo de coloração esverdeada brilhante. Os três lobos revelaram tamanhos equivalentes, não havendo uma separação segmentada entre eles.

Histologicamente, no fígado, o parênquima hepático era constituído por hepatócitos com formato que variou do arredondado irregular, hexagonal ao redondo com núcleo grande, periférico e nucléolo central. Os hepatócitos se encontravam arranjados em cordões lineares limitados por

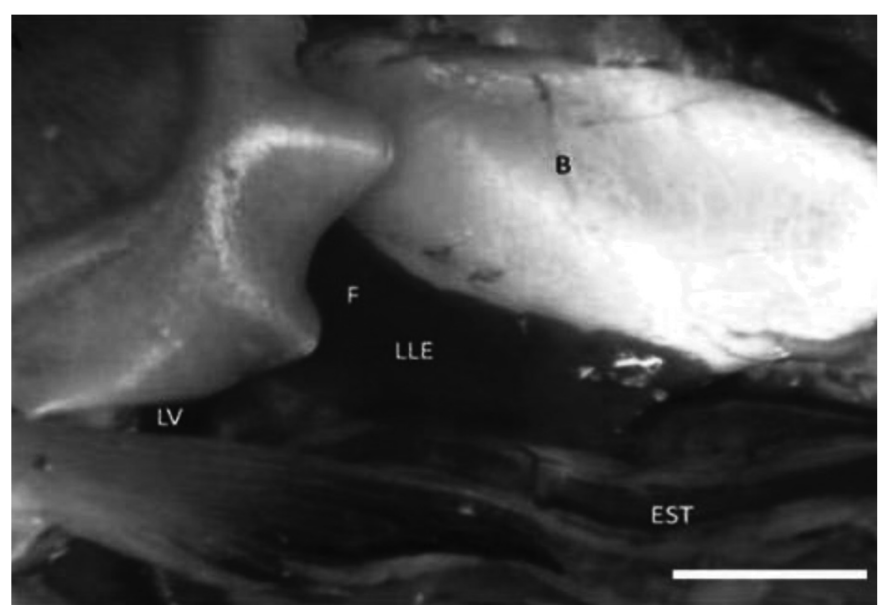

Fig.1. Vista lateral esquerda da cavidade celomática de Colossoma macropomum, evidenciando a localização do fígado (F), ventral a bexiga natatória (B), craniodorsalmente ao estômago (EST). Observar o lobo lateral esquerdo (LLE) e lobo ventral (LV) do fígado. Escala de barra: $1 \mathrm{~cm}$. 
sinusóides que irradiavam para veias centrais, observando-se em seu interior grânulos e inclusões lipídicas. As veias centrais não se localizavam no centro de unidades estruturais denominadas lóbulos hepáticos, os quais estavam ausentes no parênquima de Colossoma macropomum, não havendo uma ordem de distribuição entre as veias centrais. Em algumas regiões foram observados ductos biliares no interior de veias centrais (Fig.2).

As veias centro lobulares eram formadas por um epitélio simples pavimentoso, enquanto as artérias se apresentaram constituídas por três túnicas, a íntima, a média e a adventícia. A túnica íntima ou interna reveste internamente o vaso, constituída por uma camada de células endoteliais. A túnica média constitui-se principalmente por fibras musculares lisas, dispostas circularmente e, a túnica adventícia externamente, consistindo principalmente de tecido conjuntivo, fi-

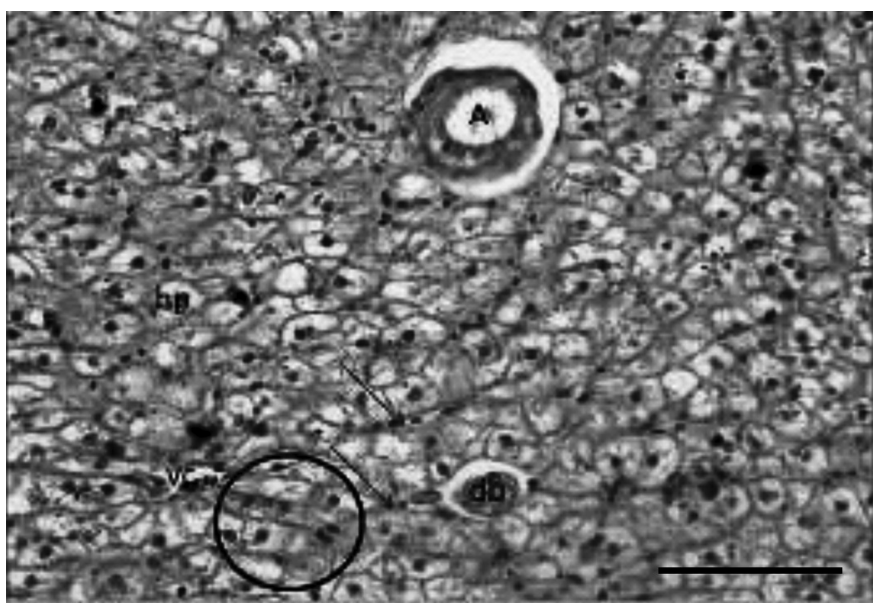

Fig.2. Parênquima hepático de Colossoma macropomum. Veia central (vc) dispersa no parênquima onde capilares sinusóides (seta) irradiam em sua direção, no interior de uma veia central ducto biliar (db). Os hepatócitos (hp), dispersos em cordões lineares (circulo) formavam o parênquima hepático. Observar formato irregular dos hepatócitos e presença de grânulos de glicogênio e inclusões lipídicas, e ausência de lóbulos hepáticos. HE, barra $=100 \mu \mathrm{m}$.

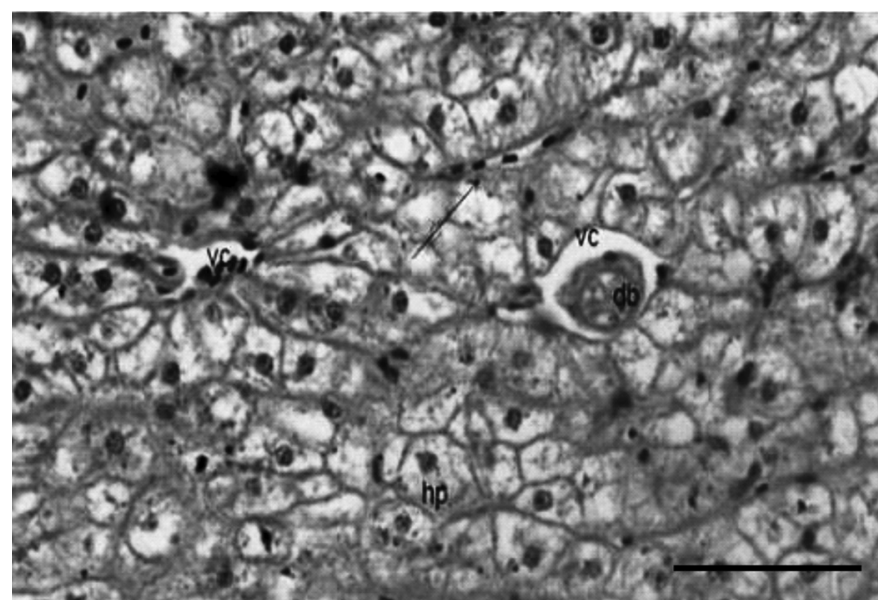

Fig.3. Parênquima hepático de Colossoma macropomum - Veias centrais (vc) e ducto biliar (db) no interior de uma veia central (vc) dispersas no parênquima constituído por hepatócitos (hp) que não apresentam forma definida. HE, barra $=100 \mu \mathrm{m}$.

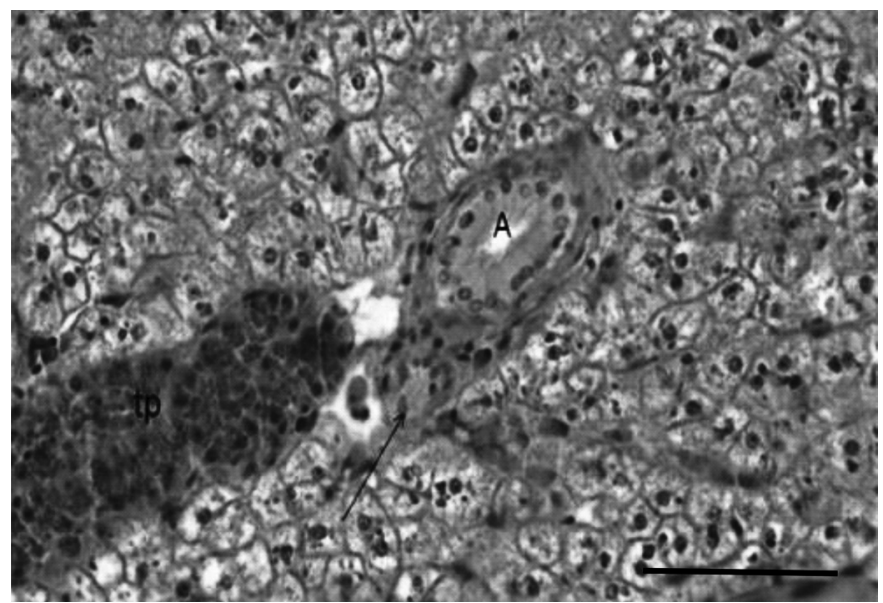

Fig.4. Parênquima hepático de Colossoma macropomum. Artéria hepática (A) associada a ducto biliar (seta) e a tecido pancreático (tp). HE, barra $=100 \mu \mathrm{m}$.

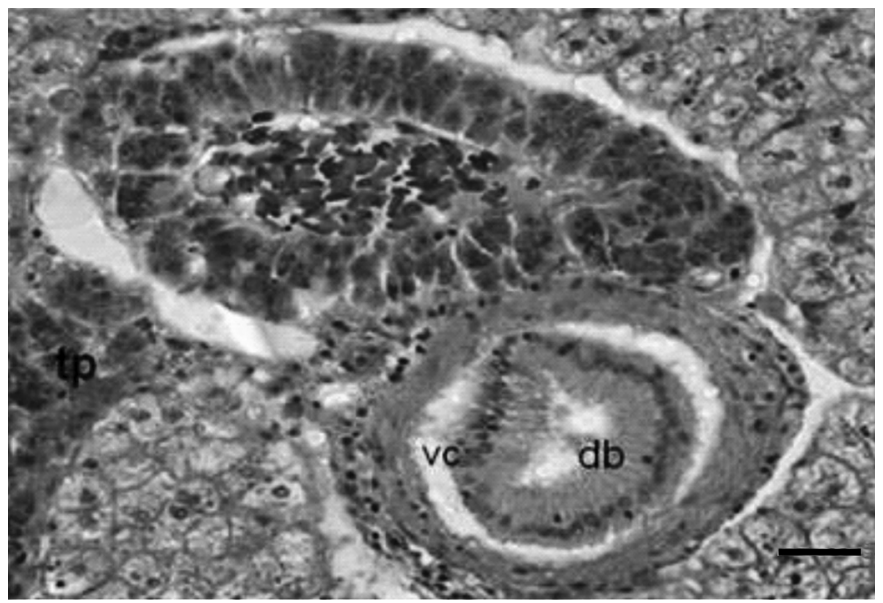

Fig.5. Parênquima hepático de Colossoma macropomum. Tríade portal formada por tecido pancreático (tp), ducto biliar (db) no interior de uma veia central (vc). HE, barra $=25 \mu \mathrm{m}$.

bras colágenas e elásticas. As artérias puderam ser observadas dispersas no parênquima, no interior de veias centrais e associadas a ductos biliares e tecido pancreático (Fig.3).

Os ductos biliares se apresentavam constituídos por um epitélio colunar prismático, com núcleos grandes, redondos, basais e basófilos, seguido por uma submucosa de tecido conjuntivo frouxo e uma camada de fibras musculares lisas. Os ductos biliares foram observados associados a uma veia central, a uma artéria, ou a tecido pancreático. Dispersos pelo parênquima pode-se observar a presença de centros de melanomacrófagos que aparentaram coloração amarelada (Fig.4).

Em Colossoma macropomum o pâncreas estava disposto de forma difusa no parênquima hepático, podendo ser observado no interior de veias centrais, ductos biliares, associados ao espaço porta ou livres, constituindo parte do estroma hepático, formando o hepatopâncreas. Este tecido formava uma estrutura semelhante a uma glândula exócrina, constituída por células ácino serosas, ricas em grânulos de zimogênio, com coloração basófila na base e eosinófila no citoplasma. 
O Espaço Porta ou Tríade Portal, na maior parte das vezes, se mostrou constituído por uma veia e um ducto biliar, duas veias e um ducto, ou uma artéria e um ducto, não tendo sido observado a formação de tríade portal usual dos vertebrados maiores (Fig.5).

\section{DISCUSSÃO}

A localização anatômica observada do fígado de Colossoma macropomum é a mesma observada em Hoplias malabaricus (Ferguson 1995) e outros peixes (Lemes 2004). Este órgão apresenta-se como um órgão compacto localizado na porção medial da cavidade celomática, com coloração vermelha amarronzado, similar ao descrito por Bruslé \& Anadon (1996), ou avermelhado (Ostrander 2000). Pode-se observar que ele apresentava adaptação ao tamanho e forma desta cavidade, e ao espaço utilizado pelos outros órgãos viscerais. Com relação ao número de lobos hepáticos, este órgão varia muito entre as espécies de teleósteos, podendo, em alguns casos não apresentar lobação reconhecida ou apresentar até três lobos bem distintos (Bruslé \& Anadon 1996). 0 fígado de tilápia do Nilo (Oreochromis niloticus) é formado por dois lobos bem definidos, apresentando o lobo esquerdo maior que o direito, o qual se estende por quase toda cavidade corpórea (Vincentine et al. 2005). 0 fígado de C. macropomum era formado por três lobos bem distintos, similar ao descrito em Leporinus macrocephalus (Bombonato et al. 2007). A vesícula biliar bem desenvolvida, de formato arredondado e se estendendo caudalmente pela cavidade celomática também foi descrita em outros peixes. (Fergunson 1995, Ostrander 2000, Fishelson 2006, Flores-Lopes \& Malabarba 2007).

Histologicamente, o padrão geral de constituição de parênquima hepático em teleósteos, apresenta hepatócitos com núcleos grandes, arredondados, localizados na região central ou basal da célula, com nucléolo proeminente, com vasos sinusóides presentes entre os hepatócitos. 0 pâncreas se encontra difuso no parênquima hepático, e este tecido é comumente encontrado junto a vasos sanguíneos, apresentando uma fina camada de tecido conjuntivo frouxo separando as células pancreáticas do parênquima hepático. As células pancreáticas organizam-se em ácinos e podem apresentar grânulos citoplasmáticos em seu interior, localizados na região apical (Flores e Malabarba 2007, Figueiredo-Fernandes et al. 2007, Bombonato et al. 2007). Estudos realizados em Leporinus macrocephalus revelaram ausência de subdivisões hexagonais, caracterizando os lóbulos hepáticos, assim como de tríades portais, estando os hepatócitos organizados em placas celulares, circundadas por sinusóides (Bombonato et al. 2007).

0 tambaqui, $C$. macropomum, apresentou este padrão de constituição de parênquima, no qual foram observadas ausência de lóbulos hepáticos e formação de tríade portal. Havia tecido pancreático formado por células cúbicas disperso pelo parênquima, normalmente próximos a um grande vaso. A presença de centros Melanomacrófagos é semelhante à descrita em muitos peixes teleósteos (Ferguson 1995).

\section{CONCLUSÃO}

A localização e as características morfológicas observadas no fígado de Colossoma macropomum são similares às descritas em outras espécies de teleósteos, diferenciando apenas no número de lobos hepáticos.

\section{REFERÊNCIAS}

Arandas J.K.G., Ludke M.C.M.M., Santos E.L., Wambach X.F., Arandas M.J.G., Lima M.R. \& Lima M.B. 2009. Análise histológica do intestino delgado da Tilápia do Nilo. IX Jornada de Ensino, Pesquisa e Extensão da UFRPE, Recife.

Arbelaéz-Rojas G.A., Fracalossi D.M. \& Fim J.I. 2002. Composição corporal de tambaqui, Colossoma,macropomum, e matrinxã, Brycon cephalus, em sistemas de cultivo intensivo, em igarapé, e semi-intensivo, em viveiros. Revta Bras. Zootec 31:1059-1069.

Bicca D.F., Querol E. \& Braccini M.C. 2006. Aspectos morfológicos e histológicos do estômago de Acestrorhynchus pantaneiro (Menezes,1992) (Teleostei, Acetrorhynchidae) na Bacia do Rio Uruguai Médio. Biodiversidade Pampeana 4:5-10.

Bombonato M.T.S., Rochel S.S., Vincentini C.A. \& Vicentini I.B.F. 2007. Estudo morfológico do tecido hepático de Leporinus macrocephalus. Acta Sci. Biol. Sci. 29(1):81-85.

Bruslé J. \& Anadon G.G. 1996. The structure and function of fish liver, p.77-78. In: Ibid. (Eds), Fish Morphology: Horizon of new research. A.A. Balkema Publishers, Brookfield, USA.

Ferguson H.W. 1995. Systemic Pathology of Fish. Library of Congress Cataloging in Publication Data, p.263.

Figueiredo-Fernandes A., Ferreira-Cardoso J.V., Garcia-Santos S., Monteiro S.M., Carrola J., Matos P. \& Fontaínhas-Fernandes A. 2007. Histopathological changes in liver and gill epithelium of Nile tilapia, Oreochromis niloticus, exposed to waterborne copper. Pesq. Vet. Bras. 27(3):103-109.

Flores-Lopes F. \& Malabarba L.R. 2007. Alterações histopatológicas observadas no fígado do lambarí Astyanax jacuhiensis (Cope, 1894) (Teleostei, Characidae) sob influência de efluentes petroquímicos. Biociências 15(2):166-172.

Goulding M. \& Carvalho M.L. 1982. Life history and management of the Tambaqui, (Colossoma macropomum, Characidae): An important Amazonian food fish. Revta Bras. Zool. 1:107-133.

Goulding M. 1993. Flood forets of the Amazon. Scient. American 268(3): 114-120.

Kock G., Triendl M. \& Hofer R. 1996. Seasonal patterns of metal accumulation in Arctic char (Salvelinus alpinus) from n oligotrophic Alpine lake related to temperature. Can. J. Fish. Aquat. Sci. 53:780-786.

Lemes A.S. \& Braccini M.C. 2004. Descrição e análise histológica das glândulas anexas do trato digestório de Hoplias malabaricus (Bloch, 1794), (Teleostei, Erythrinidae) Biodiversidade Pampeana 2:33-41.

Lowe-McConnell R.H. 2004. Estudos Ecológicos de Comunidades de Peixes Tropicais. Edusp, São Paulo. 536p.

Melo T.L., Melo C.E., Araújo N.B., Dias A.M., Santana A.O., Moreno V.A. \& Tejerina-Garro F.L. 2007. Caracterização alimentar da ictiofauna no baixo rio das Mortes, Planície do Bananal, MT. Anais VIII Congresso de Ecologia do Brasil, Caxambu, MG.

Santos G.M. \& Ferreira E.J.G. 1999. Peixes da Bacia Amazônica, p.345-373. In: Lowe-McConnell R.H. (Ed.), Estudos Ecológicos de Comunidades de Peixes Tropicais. Edusp, São Paulo.

Vari R.P. \& Malabarba L.R. 1998. Neotropical ichthyology: An overview, p.1-12. In: Malabarba L.R., Reis R.E., Vari R.P., Lucena Z.M.S. \& Lucena C. A.S. (Eds), Phylogeny and Classification of Neotropical Fishes. Edipucrs, Porto Alegre. 603p. 